Bull. Mater. Sci., Vol. 20. No. 8, December 1997, pp. 1069-1077. Printed in India.

\title{
Effect of heavy-ion irradiation on dielectric constant and electrical conductivity of doped and undoped nonlinear substance
}

\author{
K SOMASHEKHARA UDUPA*, P MOHAN RAO', \\ SRIRAMANA AITHAL ${ }^{\dagger \dagger}$, A P BHAT $^{\dagger+}$ and \\ D K AVASTHI ${ }^{+\dagger \dagger}$ \\ Department of Physics, Bhandarkar's College, Kundapur 576201 , India \\ ${ }^{+}$Department of Physics, Mangalore University, Mangalagangotri 574 199, India \\ ${ }^{\dagger \dagger}$ Department of Electronics, NSAM College, Nitte 574 110, India \\ ${ }^{+\neq}$Department of Physics, Poornaprajna College, Udupi 576101, India \\ ${ }^{t+t+}$ Nuclear Science Centre, Aruna Asaf Ali Marg, New Delhi 110067, India \\ MS received 3 December 1996; revised 25 July 1997
}

\begin{abstract}
Implantations were carried out on gel-grown potassium dihydrogen orthophosphate (KDP) and those doped with magnesium oxide (MgO) single crystals using $100 \mathrm{MeV}$ $\mathrm{Ag}^{+}$heavy-ion beam of 15 UD $16 \mathrm{MV}$ pelletron accelerator. To conduct a comparative study, measurements were carried out in the temperature range $243 \mathrm{~K}-403 \mathrm{~K}$ at frequencies ranging from $1 \mathrm{kHz}-1 \mathrm{MHz}$ on irradiated and nonirradiated nonlinear samples. It was observed that the mechanism of dielectric behaviour varied with frequency, temperature and ion irradiation. Further, implantation produced erratic variation in the conductivity both in the intrinsic and extrinsic regions, and also in the dielectric behaviour of the substance. The property of sensitive dependence on initial conditions, namely, chaos had set in after ion irradiation. However, the doping effect had not completely terminated the above transition, leading to chaos in the nonlinear medium.
\end{abstract}

Keywords. KDP; heavy-ion irradiation; implantation; dielectric constant; electrical conductivity; nonlinear crystal; property of sensitive dependence; chaos.

\section{Introduction}

Various polarization mechanisms in solids such as atomic polarization of the lattice, orientational polarization of dipoles, space charge polarizations can be understood very easily by studying the dielectric properties as a function of frequency and temperature for crystalline solids (Wagner and Hantemann 1950; Rao and Smakula 1965; Govinda and Rao 1975). These investigations help in detecting the structural transitions taking place in solids when abrupt changes in dielectric properties are observed. The profound changes which have occurred in the physical and chemical nature of a material essentially give information during its conductivity. Useful information regarding the mobility and production of lattice defects in hydrogenbonded crystals can be obtained by studying the electrical conductivity of these crystals (Glasser 1975). Ion implantation induces changes in the near-surface composition of some insulating materials. This is the cause of depletion of target ions from the implanted region (Arnold and Bordas 1977; Arnold and Peercy 1980; Mazzoldi and Miolello 1986). The ion implantation (Nadru and Wiss 1988) can exhibit instabilities with time due to proton exchange (Jackel and Rice 1984; Arnold et al 1988a, b).

\footnotetext{
*Author for correspondence
} 
The stability of the defects formed by ion implantation depends upon the ions which have entered the lattice. Apart from simple defects, other defects formed due to implantation manifest themselves in the crystals which determine a number of physical and chemical properties of the materials.

\section{Experimental}

Pure and doped KDP crystals were grown from gel method. The doped KDP single crystals were obtained with a mixture containing aqueous $2.5 \mathrm{M}$ solution of KDP and $6 \mathrm{ppm}$ solution of magnesium oxide in the ratio $2: 1$ by volume so that the $\mathrm{pH}$ of the mixture with sodium metasilicate solution of $\mathrm{sp}$. gr. 1.03 was 5.5. Good quality large-sized $40 \mathrm{~mm} \times 8 \mathrm{~mm} \times 7 \mathrm{~mm}$ single crystals were grown from an aqueous solution by slow evaporation and slow cooling technique. Absence of impurity was confirmed by chemical analysis, atomic absorption spectroscopy (AAS) and X-ray powder diffraction (XRD) patterns. From AAS study the magnesium oxide content in the doped material was estimated as $0.0467 \%$.

The pure and doped samples were then exposed to $100 \mathrm{MeV} \mathrm{Ag}^{+}$heavy ion beam of 15 UD pelletron accelerator at Nuclear Science Centre (NSC), Delhi. The irradiation was carried out with the beam current of 8 nano amperes for different duration, totally up to $40 \mathrm{~min}$. The samples were then annealed for $30 \mathrm{~min}$ at a suitable temperature.

The irradiated and unirradiated samples were cut by a wet string and the opposite faces were coated with good quality silver paste to obtain a good ohmic contact. Prior to the measurements, the crystals were heated and cooled to avoid surface moisture in order to get reproducible results. The samples were then kept inside a thermo stream system model TP $0412 \mathrm{~A}$ which could be maintained at any temperature between $203 \mathrm{~K}$ and $473 \mathrm{~K}$. The leads of the capacitors were then connected to the respective terminals of a Hewlett Packard Precision LCR meter model 4284 A which could operate from $20 \mathrm{~Hz}$ to $1 \mathrm{MHz}$ range. The measurements were made at various frequencies ranging from $1 \mathrm{kHz}$ to $1 \mathrm{MHz}$ and temperature from $243 \mathrm{~K}$ to $403 \mathrm{~K}$. Each time the dielectric capacitance $C_{\mathrm{p}}$ and dielectric conductance $G$ values were noted down from the display screen when the readings were steady.

The measured values of capacitance at different frequencies and temperatures were converted into dielectric constant $(K)$ using the relation

$$
K=C \cdot d / A \varepsilon_{0},
$$

where $C$ is the capacitance of the sample, $d$ the thickness of the sample, $A$ the area of the face in contact with the electrode and $\varepsilon_{0}$ the permittivity of free space. Thus the values of dielectric constant were determined as a function of different frequencies and temperatures. However the measured values of the conductance $G$ at different frequencies and temperatures were converted into electrical conductivity by using the relation

$$
\sigma=G \cdot d / A
$$

where $G$ is the measured conductance, $d$ the thickness of the sample and $A$ the area of the face in contact with the lead. The $\sigma$ values were fitted into the equation

$$
\sigma T=\sigma_{0} \exp (-E / K T),
$$

and the activation energy $(E)$ values were calculated. 


\section{Results and discussion}

Figures 1-4 show the plots of dielectric constant against temperature at constant frequency for KDP and doped KDP before and after implantation with heavy $\mathrm{Ag}^{+}$ion beam from pelletron accelerator, respectively. It is seen that dielectric constant decreases with the increase of temperature at all frequencies as in figure 1 . There is an appreciable increase in the value of $K$ after doping and $K$ decreases with the increase of frequency as seen in figure 2 . The dielectric constant of a material is generally composed of four types of contributions, viz. ionic, electronic, orientational and space charge polarizations. All these may be active at low frequencies. The nature of variations of dielectric constant with frequency and temperature indicates the type of contributions that are present in them. The dipolar orientational effect can be seen in some materials at high frequencies and ionic and electronic polarizations below $10^{3} \mathrm{~Hz}$. The large value of $K$ at low frequency and at low temperature is due to the presence of space charge polarization (Suryanarayana et al 1984), which depends on the purity and perfection of the sample. At low frequency and at low temperature range it is noticeable.

Temperature has a complicated influence on the dielectric constant. The decrease in $K$ with the increase in temperature in KDP and doped KDP crystals may be due to

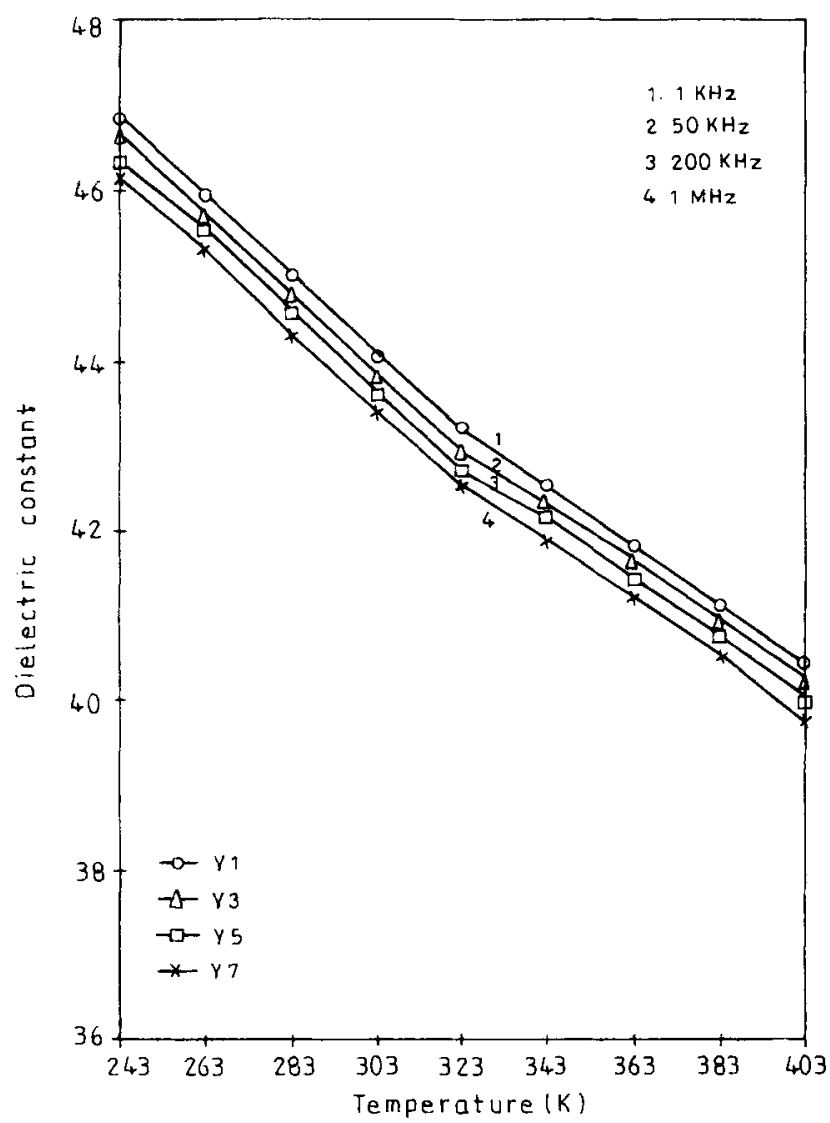

Figure 1. Variation of dielectric constant with temperature at constant frequency for KDP crystals along $\mathrm{b}$ axis. 


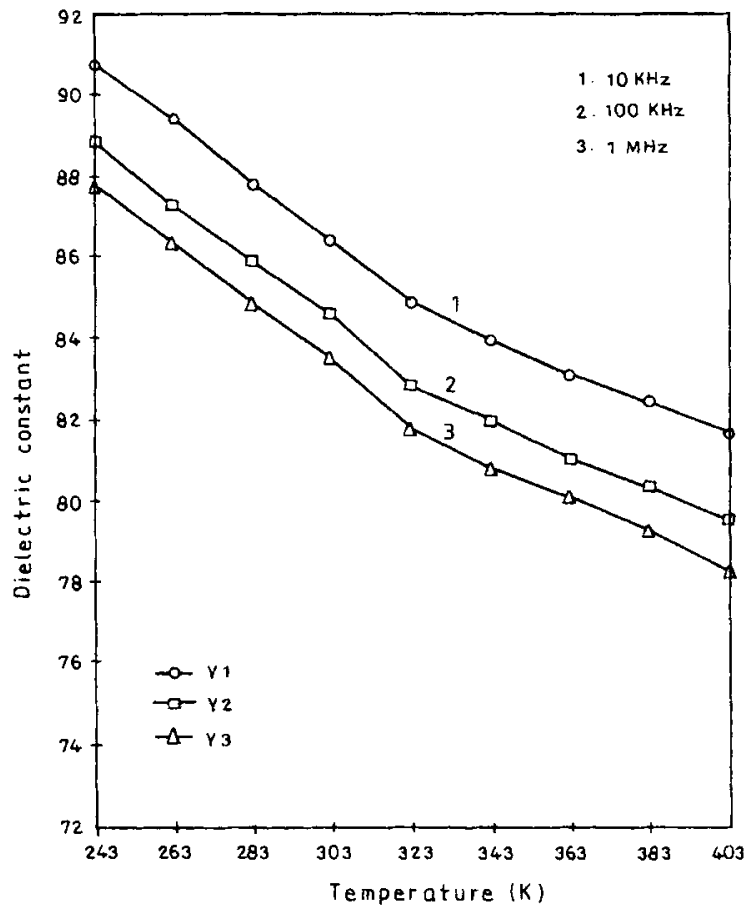

Figure 2. Variation of dielectric constant with temperature at constant frequency for doped $\mathrm{KDP}$ crystals along $\mathrm{b}$ axis.

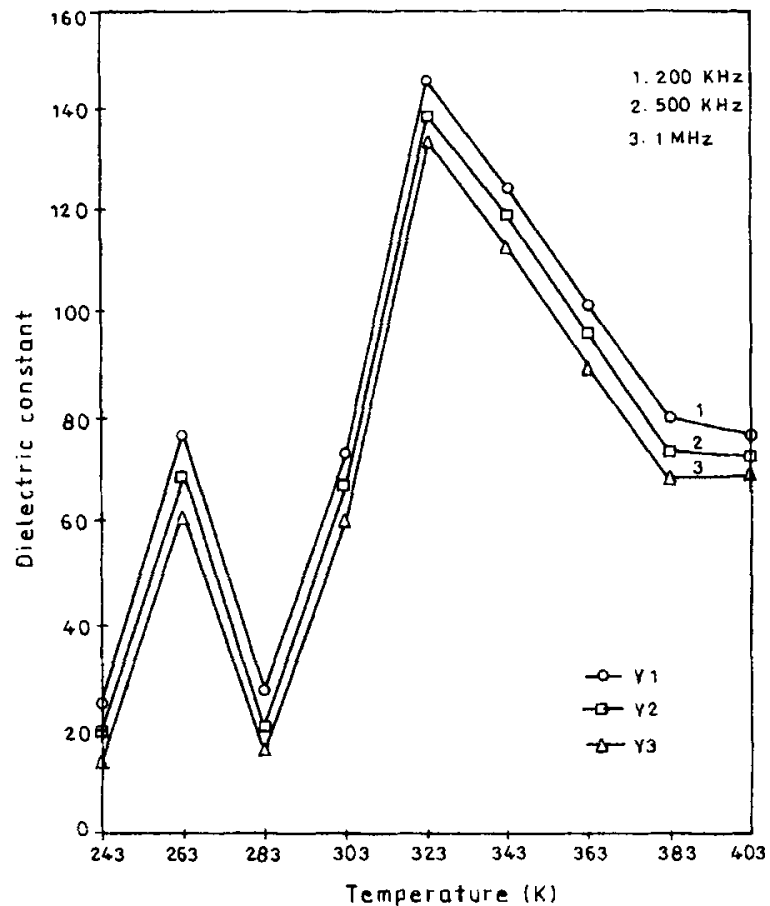

Figure 3. Variation of dielectric constant with temperature at constant frequency for irradiated KDP crystals along the $\mathrm{b}$ axis. 


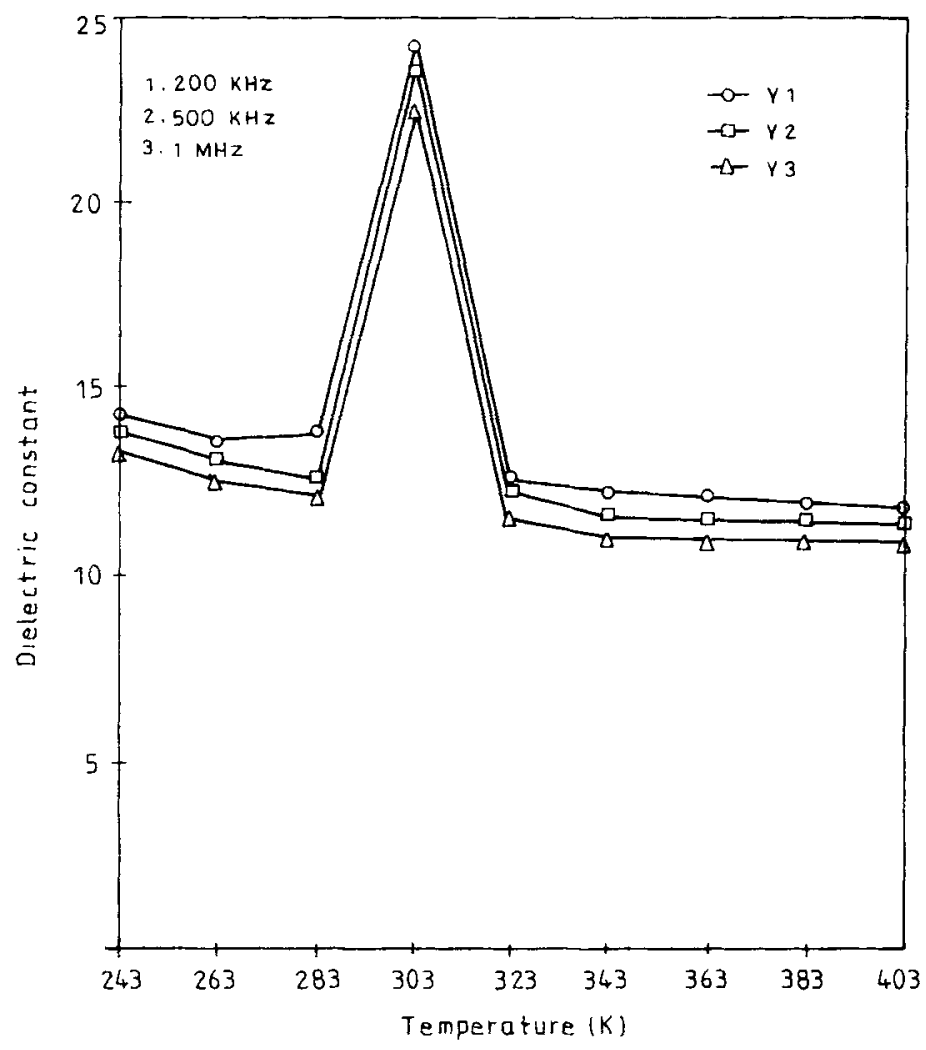

Figure 4. Variation of dielectric constant with temperature at constant frequency for irradiated doped KDP crystals along $b$ axis.

dehydration process of water molecules. There is ion dipole interaction between dipole moments of water molecules and the effective lattice charges. Thermal energy makes the water molecules free and finally dehydration of these molecules will take place as temperature increases.

Figures $5-8$ show the plots of $\log _{10}(\sigma T)$ against $1 / T 10^{3}\left(K^{-1}\right)$ for pure, doped KDP and the same after implantation with heavy $\mathrm{Ag}^{+}$ion beam when the applied electric field is perpendicular to the $\mathrm{C}$ axis. The conductivity graphs exhibit the usual intrinsic and extrinsic regions except in figure 7 . The intrinsic conduction region seems to be connected to mobility of vacancies. The low activation energy suggests that oxygen vacancies may be responsible for conduction in this region. In KDP type of crystals the possible type of point defects which help the electrical conduction process are the ionization defects, viz. $\left(\mathrm{HPO}_{4}\right)^{2-}$ and $\left(\mathrm{H}_{3} \mathrm{PO}_{4}\right)$ produced as a result of proton jump from one phosphate group to another along the same bond (George 1989) and $L$ and $D$ defects.

The impurities and vacancies are weakly attached in the lattice and are formed due to the presence of ions of other valency. They can easily migrate within an external electric field since the activation energy required for their drift is rather low. The presence of impurities and vacancies mainly determine the region of so-called low temperature conductivity of the crystal. The energy needed to form the defect is much larger than the 


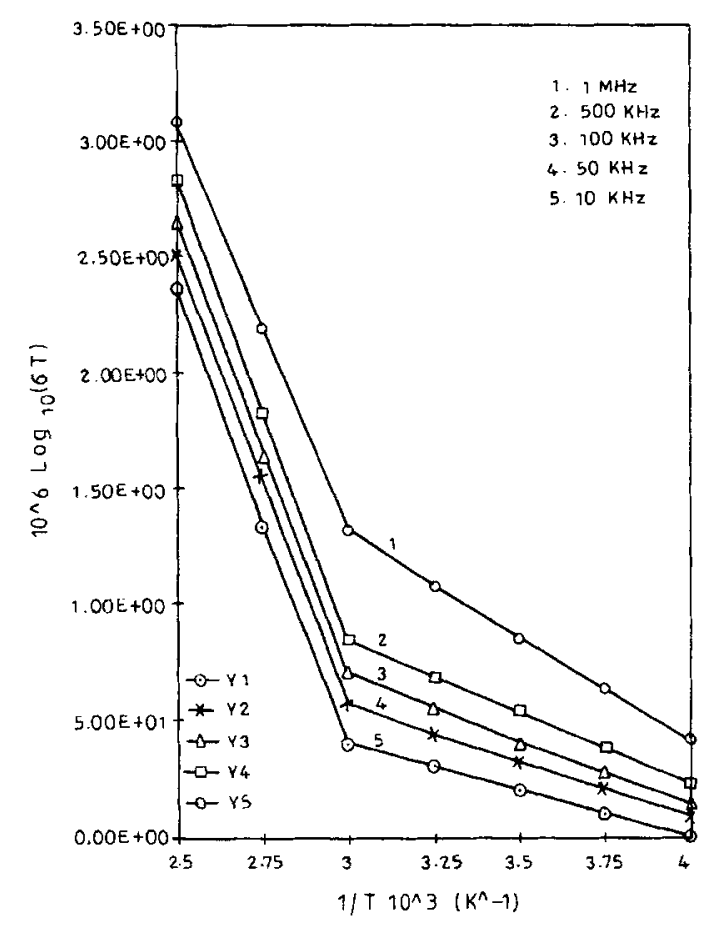

Figure 5. Variation of $\log _{10}(\sigma T)$ with $1 / T 10^{3}$ at constant frequency for KDP crystals along $\mathrm{b}$ axis.

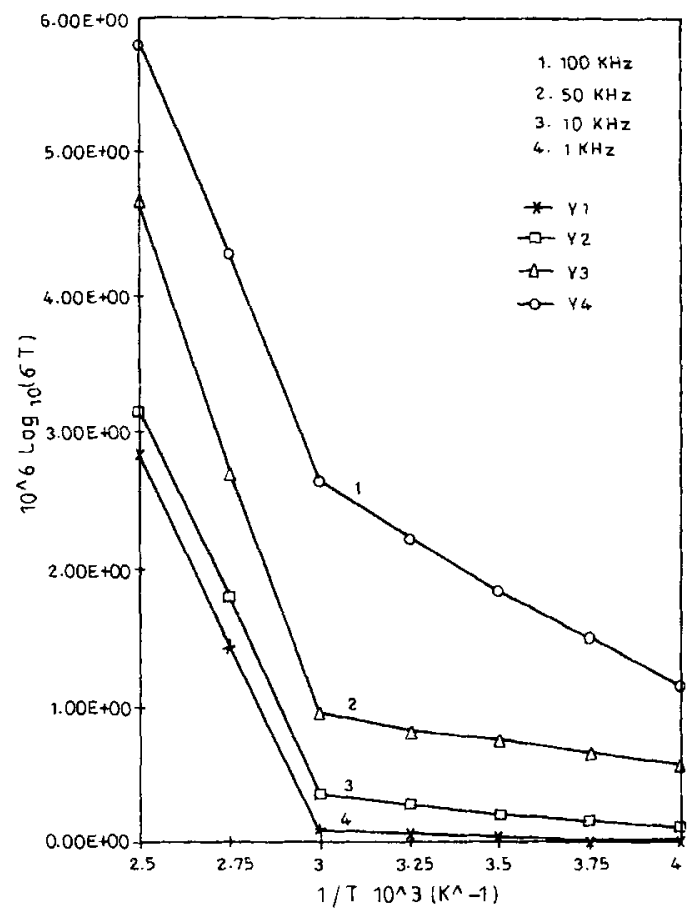

Figure 6. Variation of $\log _{10}(\sigma T)$ with $1 / T 10^{3}$ at constant frequency for doped KDP crystals along $b$ axis. 


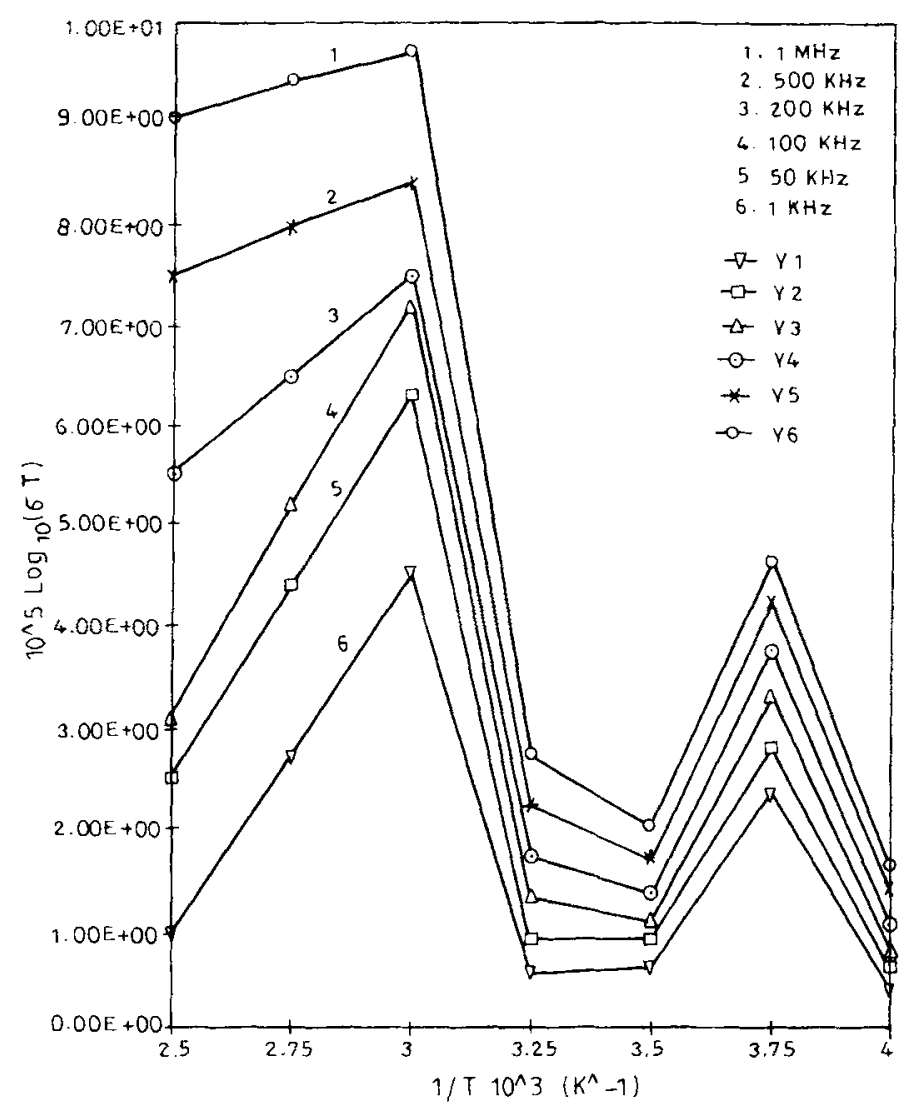

Figure 7. Variation of $\log _{10}(\sigma T)$ with $1 / T 10^{3}$ for irradiated KDP crystals along b axis.

energy needed for its drift. The intrinsic defects caused by the thermal fluctuations in a crystal determine the conductivity of the crystal at higher temperature.

Figure 1 shows almost steady variation representing the usual behaviour found in pure KDP crystals. A smooth variation of $K$ in figure 1 has developed into 4 fragments in figure 3. Figure 3 shows the system approximately generating the step 3 (figure 9) of the Koch curve starting from a line segment in figure 1. It is an example for a fractal. It is the first step leading transition to chaos (Gulick 1992). However, the irradiated doped KDP (figure 4), in trying to stabilize this effect started generating the initial step 2 (figure 9) of the Koch curve (Benoit 1982) from a near-line segment shown in figure 2. They are again fractals leading transition to chaos.

Figure 7 shows the conductivity variation of pure KDP after the ion irradiation. From figures 5 and 7 it can be seen that the electrical conductivity has increased after ion irradiation even though the variations are erratic in figure 7 . The intrinsic and extrinsic regions are linear in figure 5 and they are nonlinear in figure 7 . The system has become complex after ion irradiation and it shows irregular behaviour or randomness. It means deviation from the conception of continuity and order and generally nonlinearity. Figure 7 shows the intrinsic and extrinsic regions generating the initial steps (figure 9) of the Koch curve starting from line segments in figure 5. It is a good 


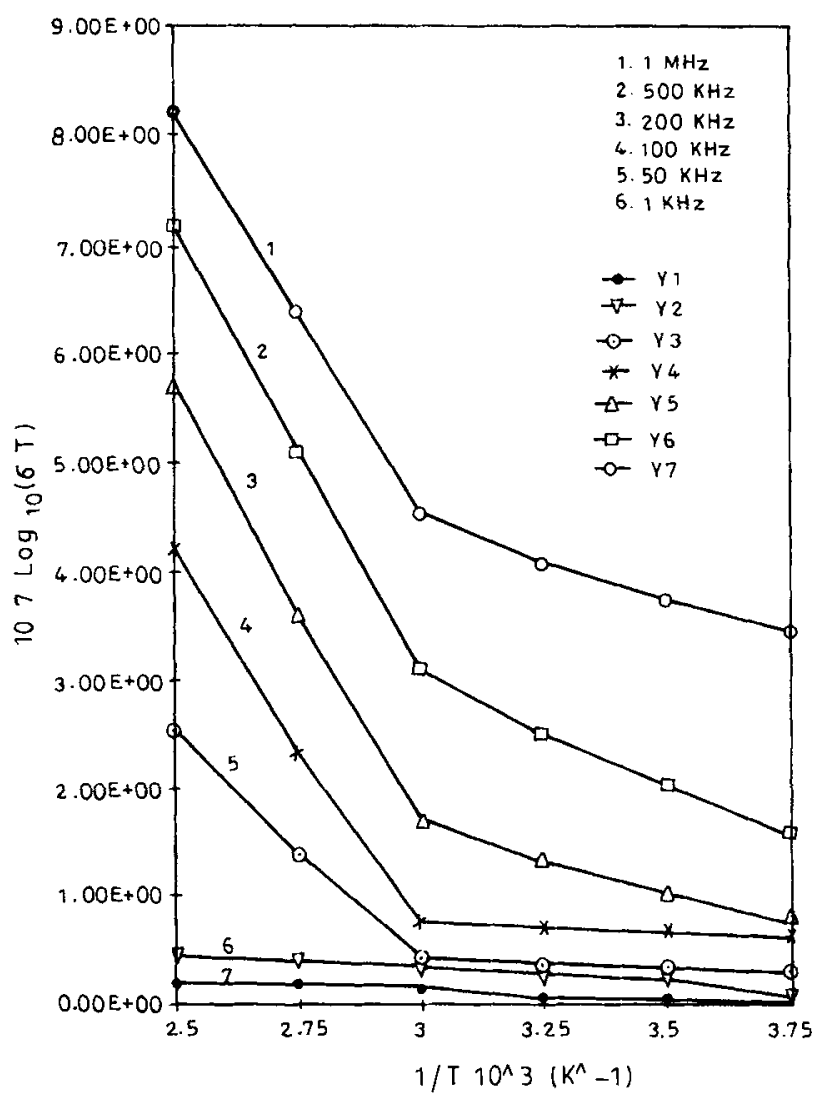

Figure 8. Variation of $\log _{10}(\sigma T)$ with $1 / T 10^{3}$ for irradiated doped KDP crystals along $\mathrm{b}$ axis.

example for fractal. It is the first step exhibited in the nonlinear medium leading transition to chaos (Gulick 1992).

The property of sensitive dependence on initial conditions is one of the characteristics of transition leading to chaos. This property has set in only after ion irradiation. A nonlinear medium may be susceptible to transition leading to chaos after ion irradiation. However in figure 8 , it is seen that the irradiated doped crystal has almost retained its electrical conductivity after the range of $50 \mathrm{kHz}$. It explains that the doping effect here has cut off the sensitive dependence on initial conditions, viz. chaos in the nonlinear medium.

The advent of chaos introduces a new type of attractor called strange attractor or chaotic attractor. Geometrically a strange attractor is a fractal. Fractals describe the phase space portrait of chaotic systems. Koch curve is a fractal. The strange attractors and hence chaotic behaviours are found in certain nonlinear dynamical systems. The present system is made a dynamical system by assigning frequency at all stages of its observations. The Koch curve can be constructed as follows: A line segment (figure 9, step 1) is taken. It is then split up into three parts. The middle one third is replaced by two line segments (figure 9, step 2). The above operation is performed on the four line segments (figure 9, step 3). This process is repeated infinite times to get the final Koch curve. The actual Koch curve could not be seen here because of the following reasons 


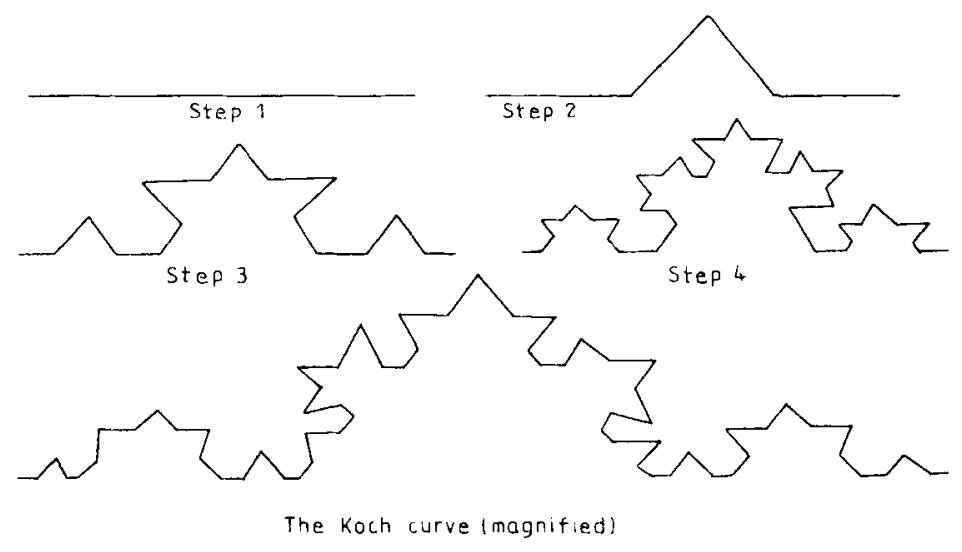

Figure 9. Generating the Koch curve starting from a line segment.

namely: (i) the initial line was inclined to the $X$-axis, (ii) the computer adopted different $\mathrm{X}$ and $\mathrm{Y}$ scales, (iii) the original line was deviated from a line segment, and (iv) the observations were made only on limited points etc.

\section{Conclusions}

The property of sensitive dependence on initial conditions namely chaos had set in after ion irradiation in the nonlinear medium as seen in figures 3,4 and 7. Doping such a nonlinear material with $\mathrm{MgO}$ could not terminate the effect of transition leading to chaos but reduced the effect to a large extent as seen in figures 4 and 8 . Nonlinear substances may be susceptible to transition leading to chaos after long time irradiation of 8 nano ampere of current totally for 40 minutes and more.

Chaos can be an eventuation spaced out in a span of time from orderliness to randomness, or from logically definable to logically incomprehensible in reference to the characterizing parameters of the initial state.

\section{References}

Arnold G W and Bordas J A 1977 J. Appl. Phys. 481483

Arnold G W and Peercy P S 1980 Non. Cryst. Solids 41359

Arnold G W. Battaglin G, Della Mea G and Miotella A 1988a Nucl. Instrum. \& Meth. B32 315

Arnold G W, Carnera A and Mazzoldi $P$ 1988b Laser and particle heam chemical processing for microelectronics (eds) D J Ehrlich, G S Higasiu and M M Oprysko (Pittsburgh: Materials Research Society) p. 453

Benoit B Mandelbrot 1982 The fractal geometry of nature (San Francisco: Freeman)

George A W 1989 Nucl. Instrum. \& Meth. Phys. Res. B39 708

Glasser 1975 Chem. Rev. 7521

Gulick D 1992 Encounters with chaos (New York: McGraw Hill)

Govinda S and Rao K V 1975 Phys. Status Solidi 27639

Jackel J L and Rice C E 1984 Proc. SPIE $\mathbf{4 6 0} 43$

Mazzoldi P and Miolello A 1986 Radiat. Eff. 9839

Nadru J M and Wiss B L 1988 J. Light wave Technol. LT T-4, B32 315

Rao K V and Smakula A A 1965 J. Appl. Phys. 362031

Robert May 1976 Nature 261985

Suryanarayana P, Acharya H N and Rav K 1984 J. Mater. Sci. Lett. 321

Wagner G and Hantemann P $1950 \mathrm{~J}$. Chem. Phys. 1872 